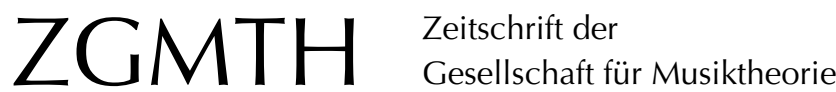

Diergarten, Felix (2016): Klaus-Jürgen Sachs, Musiklehre im Studium der Artes. Die Musica (Köln 1507) des Johannes Cochlaeus (= Veröffentlichungen des Staatlichen Instituts für Musikforschung, Bd. 21; Studien zur Geschichte der Musiktheorie, Bd. 11), Hildesheim: Olms 2015. ZGMTH 13/1, 157-161. https://doi.org/10.31751/881

(C) 2016 Felix Diergarten

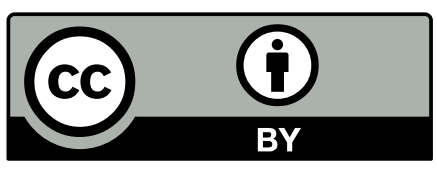

Dieser Text erscheint im Open Access und ist lizenziert unter einer Creative Commons Namensnennung 4.0 International Lizenz.

This is an open access article licensed under a

Creative Commons Attribution 4.0 International License.

eingereicht / submitted: 05/01/2017

angenommen / accepted: 10/01/2017

veröffentlicht / first published: 20/02/2017

zuletzt geändert / last updated: 03/08/2018 


\section{Klaus-Jürgen Sachs, Musiklehre im Studium der Artes. Die Musica (Köln 1507) des Johannes Cochlaeus (= Veröffentlichungen des Staatlichen Instituts für Musikforschung, Bd. 21; Studien zur Geschichte der Musiktheorie, Bd. 11), Hildesheim: Olms 2015}

Wenige haben die Forschung zur Geschichte der spätmittelalterlichen und frühneuzeitlichen Musiktheorie so substantiell bereichert wie Klaus-Jürgen Sachs. Seine Freiburger Dissertation Der Contrapunctus im 14. und 15. Jahrhundert, die 1974 in den Beiheften zum Archiv für Musikwissenschaft erschien und dann zu einem Kapitel für Band 5 der von Frieder Zaminer herausgegebenen Geschichte der Musiktheorie umgearbeitet wurde, stellt noch immer das internationale Referenzwerk zur Thematik dar. ${ }^{1}$ Detaillierte Kenntnisse der Geschichte der Musiktheorie im Allgemeinen und der Satz- und Kompositionslehre im Besonderen stellte Sachs daraufhin auch in zahlreichen Artikeln unter Beweis, die von der unermüdlichen Bereitschaft geprägt sind, einer komplexen Materie in klarer und unprätentiöser Sprache zur anschaulichen Darstellung zu verhelfen. Dazu gehören (um nur wenige herauszugreifen) die »Kontrapunkt«-Artikel im Handwörterbuch der musikalischen Terminologie, in The New Grove und im Riemann-Lexikon, der Beitrag zum Artikel »Komposition« in Die Musik in Geschichte und Gegenwart (2. Auflage), der Artikel "Musikanschauung, Musiklehre, Musikausbildung" im Band Die Musik des 15. und 16. Jahrhunderts des Neuen Handbuch der Musikwissenschaft sowie das Kapitel "Musikalische Elementarlehre im Mittelalter in Band 3 der Geschichte der $\mathrm{Mu}$ siktheorie. 2002 ergänzte Sachs seine Monographie zum Contrapunctus mit den Studien zu musikalischen Lehrtexten des späten 15. Jahrhunderts, die einige in der früheren Studie ausgelegte Fäden aufgreifen und anhand von

1 Sachs 1974 und 1984.

2 Ein Schriftenverzeichnis findet sich in Kleinertz/Flamm/Frobenius 2010, 607-617. drei im Rahmen der Studie erstmals edierten musiktheoretischen Quellen ein neues Bild von der mehrstimmigen Kompositionslehre vor 1500 zeichnen. $^{3}$

Nun hat Klaus-Jürgen Sachs erneut an vorausgehende Arbeit angeknüpft und einmal mehr einen großen Forschungsbeitrag geleistet, der mit der Musica des Johannes Cochlaeus (Köln 1507) eine weithin bekannte, aber bislang schwer zugängliche Quelle im Kontext weiterer Dokumente und einer umfassenden Studie zur Musiklehre im Studium der Artes vorlegt. Die Herausgeber der Reihe Studien zur Geschichte der Musiktheorie kündigen den Band in ihrem Vorwort als »musiktheoretische Preziose« (vii) an. Und sie haben Recht, denn verschiedene Umstände machen die Auseinandersetzung mit Cochlaeus' $\mathrm{Mu}$ sica so schwierig wie aufschlussreich. Da ist zum einen der Umstand, dass der Traktat in vier verschiedenen Fassungen vorliegt. Die ersten beiden, kürzeren Vorläuferfassungen sind anonym überliefert (werden heute aber Cochlaeus zugeschrieben), die dritte ist die zentrale Kölner Musica von 1507, die vierte schließlich eine gekürzte Neuauflage der letzteren. Hinzu kommt die Tatsache, dass die Musica eine jener zeittypischen Kompilationen ist, bei denen direkte Zitate und sinngemäße Entlehnungen aus zahlreichen Quellen auf der einen Seite mit eigenen Hinzufügungen und Erweiterungen des Autors und Kompilators (bzw. mit noch nicht identifizierten Entlehnungen) auf der anderen Seite teilweise schwer entwirrbar verknotet sind. Zu dieser komplexen Gemengelage tritt bei Cochlaeus' Musica schließlich noch der glückliche Umstand, dass in einigen überlieferten Exem-

3 Sachs 2002. 
plaren des Drucks handschriftliche Glossen von Benutzern enthalten sind, die den Text des Cochlaeus erläutern, umschreiben oder kritisieren. Einer dieser Benutzer war kein Geringerer als der Cochlaeus-Schüler Heinrich Glarean, der als Schreiber zahlreicher und aufschlussreicher Glossen der Forschung bereits bekannt ${ }^{4}$, dessen Anmerkungen zur Cochlaeus-Lehre aber (einschließlich eines Lobgedichts auf den Lehrer) hier erstmals zugänglich gemacht sind.

Klaus-Jürgen Sachs stellt sich der anspruchsvollen Aufgabe, das Gewirr von Entlehnungen, Fassungen und Glossen nicht nur les- und nutzbar zu machen, sondern auch in einem reichen Kontext zu verorten. Das auf zwei Bände aufgeteilte Werk umfasst lateinischen Text und Übersetzung der Musica inklusive zweier dazugehöriger, von Cochlaeus selbst verfasster pädagogischer Exzerpte (ebenfalls mit Übersetzung). Die Edition bietet weiterhin einen ausführlichen chronologischen Kommentar zu den einzelnen Kapiteln der edierten Quellen, eine Einführung in Cochlaeus' Leben und Werk im Zusammenhang der Artes-Lehre und eine Transkription ausgewählter Glossierungen (ohne Übersetzung), wobei die Glossen Glareans vollständig, weitere Glossen »nur auszugsweise für bestimmte Erörterungsziele« (xii) wiedergegeben sind. Zwar bringt ein solch ambitioniertes Unterfangen eine gedruckte Edition an die Grenzen des Machbaren und zwingt zu "pragmatisch-vorläufigen Maßnahmen« (xii), doch darf man sagen, dass hier das Machbare in vorbildlicher Weise gelungen ist. Die Aufteilung in zwei Bände, deren erster lateinisches Original und deutsche Übersetzung auf gegenüberliegenden Doppelseiten, deren zweiter Kommentar und Glossen enthält, macht es möglich, mit zwei aufgeschlagenen Büchern dem Quellentext und dem Kommentar parallel zu folgen. Lediglich das Nachschlagen in den Glossen erfordert also das Blättern im Kommentarband, das aber durch die vom Herausgeber eingefügten Gliederungszeichen

4 Vgl. Fenlon 1994; Judd 2000, 124f.; Forscher Weiss 2010; Mai Groote 2013. erleichtert wird. Edition und Übersetzung des Quellentextes sind so gestaltet, dass sie tatsächlich als Fließtext »les- und benutzbar" (51) sind. Dazu ist der textkritische Apparat jeweils an das Ende der (kurzen) Kapitel gestellt und auf das Sinnvolle beschränkt. Abweichungen der Vorläuferfassungen vom Haupttext der Kölner Musica von 1507 sind nur da vermerkt, wo sie Signifikantes, nicht aber Beiläufiges oder Detailspezifisches betreffen. Die gute Lesbarkeit ist hier dem Herausgeber Sachs und seinem Mut zu verdanken, in dieser Sache Entscheidungen pragmatisch zu treffen, obgleich natürlich »keine zweifelsfreie Grenze gezogen werden « (51) kann. Ebenso an die Kapitelenden gestellt sind Hinweise auf die zahlreichen Entlehnungen aus den Schriften von Franchinus Gaffurio, Adam von Fulda, Melchior Schanppecher und vielen anderen. Die Musikbeispiele sind auf der Übersetzungsseite (wo sinnvoll) im modernen Notensatz wiedergegeben, auf der lateinischen Seite faksimiliert, sodass zur Sichtung der originalen Notenbeispiele das Nachschlagen im Faksimile nicht notwendig ist. Ein terminologisches Verzeichnis lateinischer Fachtermini sowie ein Personen- und Sachregister runden das Ganze ab.

Die nun vorliegende Edition bietet eine umfassende Materialschau zur Musiklehre um 1500, und zwar anhand eines besonders eindrucksvollen Beispiels, dessen Autor bei der Lektüre in seiner Rolle als Lehrer im humanistischen und universitären Umfeld des frühen 16. Jahrhunderts geradezu mit Händen greifbar wird. Im Luther-Jahr 2017 dürfte der Name des Johannes Dobeneck (der sich nach seiner Heimatpfarrei Wendelstein latinisiert Cocleus oder Cochlaeus nennen ließ) wohl vor allem in Zusammenhang mit seiner Rolle als theologischer Gegner Luthers und als Verfasser antireformatorischer Streitschriften fallen, die jahrhundertelang das Bild des Reformators aus katholischer Sicht bestimmten. Tatsächlich beschränkt sich Cochlaeus' Aktivität als Musiklehrer und Musikpublizist offenbar auf einen kurzen Abschnitt seines Lebens, nämlich auf seine Zeit als Schüler und Magister an der Universität Köln und als LorenzschulRektor in Nürnberg, eine nur knapp zehn 
Jahre umfassende, aber ungemein produktive Phase, in der Cochlaeus die sechs Texte seiner Musiklehre »in relativ kurzen Abständen und offenbar rasch zum Druck gab« (45f.).

Ob die nun vollständig in Editionen vorliegenden Schriften des Cochlaeus (sein Nürnberger Tetrachordum war schon länger greifbar ${ }^{5}$ ) tatsächlich zu einer "Neubewertung" (vii) der Rolle der Musiklehre im Kontext der Artes führen wird, wie die Reihenherausgeber sich erhoffen, muss sich zeigen. Schließlich war Cochlaeus' Text und sein universitäres Umfeld bis anhin zwar schwieriger zugänglich, aber nicht unbekannt. In jedem Fall aber wirft die Art und Weise, wie Cochlaeus in seiner Musica »theoretisches" und "praktisches Lehrgut" (14) mischt, ein interessantes Licht auf die universitäre und schulische Musiklehre des frühen 16. Jahrhunderts, denn Cochlaeus' Musica beschränkt sich eben nicht auf »rein theoretisches" oder »spekulatives" Gedankengut: Sie umfasst auch Anweisungen der praktischen Musik bis hin zur ersten vierstimmigen Kadenzlehre der Musikgeschichte. „Die Zugehörigkeit der musica zu den Artes liberales« bleibt dabei aber (einer älteren musikwissenschaftlichen Auffassung zum Trotz) eine »weithin selbstverständliche Anschauung« (19). Die Stellung der universitären $m u$ sica, das lässt sich nun der Neu-Edition hervorragend entnehmen, war zum einen durch ihren »quadrivialen Kern" als »eine der zahlengegründeten (`mathematischen`) Artes« gekennzeichnet, zum anderen aber eben auch durch die Erfordernisse der »alltagsüblichen und zeremoniell erforderlichen musikalischen Praxis» (19).

Cochlaeus' Musica besteht aus drei Büchern und folgt mit dieser Gliederung einschlägigen Vorbildern. Das erste Buch umfasst die Elementar- und Chorallehre, also die Lehre vom Tonsystem, der Solmisation, der Tonarten und Psalmtöne, das zweite Buch umfasst die Mensurallehre, das dritte schließlich die Satzlehre, die bei Cochlaeus als "ars componendi« bezeichnet wird. Sie enthält zahlreiche Regeln und Anleitungen für den »compositor« (172),

5 Cochlaeus 1970.
Regeln, die zwar weitgehend aus anderen Quellen (insbesondere aus der Practica Musicae des Gaffurio) entlehnt sind, im Einzelnen aber einen »klug kompilierenden« (329) Autor zeigen. Als einer der wenigen wirklich originalen Beiträge des Cochlaeus muss (bis auf Weiteres) seine vierstimmige Kadenzlehre gelten. ${ }^{6}$ Diese »Früh(est)-Belege« (328) der mehrstimmigen Kadenzlehre machen die $\mathrm{Mu}$ sica zu einem für die Geschichte der Satzlehre zentralen Dokument und spiegeln (gemeinsam mit den von Sachs bereits edierten Regensburger Traktaten ${ }^{7}$ ) »jene Entwicklungsphase wider, in der die Contrapunctuslehre mühsam und vorsichtig, doch zwangsläufig in Richtung einer umfassenderen Ars componendi zu erweitern oder umzugestalten war" (327). Eine musikhistorisch mehrschichtige Trouvaille ist dabei eine handschriftliche Glosse Glareans, in der dieser einem zweistimmigen Kontrapunkt-Beispiel, das Cochlaeus von Gaffurio entlehnt, eine dritte Stimme als Unterstimme hinzufügt und dabei an einer Stelle offenbar Oktavparallelen zwischen Tenor und Contratenor bassus, hier als "vox gravis« bezeichnet, in Kauf nimmt (175).

Das herausragende und originelle Kennzeichen der Schriften des Cochlaeus jedoch, das hier Gelegenheit zu einem abschließenden Ausblick in die Gegenwart gibt, ist ihre pädagogische Ausrichtung. Die gesamte gedruckte Musiklehre des Cochlaeus steht in unmittelbarem Zusammenhang mit seiner Tätigkeit als Lehrer an Universität und Schule. An vielen Einzelheiten zeigt sich, dass Cochlaeus "eine besonders klare, einprägsame Darstellung seiner Lehre« anstrebte, was sich insbesondere an sprachlichen Kürzungen gegenüber den herangezogenen Quellen und an seinem Bemühen zeigt, »alle leitenden Fachausdrücke zu erklären« sowie »sinnfällige Diagramme und Notenbeispiele zur Veranschaulichung" einzufügen (42). Doch mehr als das: Cochlaeus ergänzte seine Musica mit zwei eigens im Druck erschienenen Begleittexten, die jeweils nur acht Seiten umfassen und offenbar einen

6 Vgl. dazu auch Schwind 2009, 135-139.

7 Sachs 2002. 
gewissen »Nachhilfe-Effekt« (36) beabsichtigten. Sie stellen das Wesentliche der Lehre knapp rekapitulierend (und wohl auch zur Prüfungsvorbereitung gedacht) zusammen. Im Vorwort des ersten dieser beiden NachhilfeTexte beschreibt Cochlaeus, dass die Kunstfertigkeit (»ars») sich bekanntlich ohnehin schon mit »recht Schwierigem« beschäftige, dass diese Dinge aber dem unwissenden Anfänger manchmal sogar "äußerst schwierig" erschienen (201). ${ }^{8}$ Er empfiehlt daraufhin den Lehrenden (»docentes«), zum Nutzen der Schüler »Verschiedenstes aus[zu]probieren, gemäß den verschiedenen und ungleichen geistigen Fassungskräften « (201) der Schüler. ${ }^{9}$ Cochlaeus entwirft »Übungen« (»exercitium«) für ganz elementare Aspekte des Lesens und Singens, »um Ärger zu vermeiden, damit nicht etwa im Chor stumm herumgestanden oder, wenn man gegenüber anderen falsch singt, das Gehör der Umstehenden beleidigt wird « (201). ${ }^{10}$ Für solche Bedürfnisse des Alltags an Universität und Schule war Cochlaeus offenbar bereit, das theoretische Hintergrundrauschen bisweilen auf ein Mindestmaß zu reduzieren. Allerdings nur in der Darstellung, nicht in der Sache, denn die Basislehre würde Luftwurzeln treiben ohne die großen Theorien im Hintergrund, an die sie in jedem Detail zurückgebunden ist. Der Unterschied zwischen den verschiedenen Teilbereichen der Musiklehre, so könnte man sagen, ist kein grundsätzlicher und inhaltlicher, er ist ein institutioneller und sprachlicher: Welche Aspekte des weiten Feldes musikalischer Lehre an welchem Ort und welchem Zeitpunkt der Ausbildung in welcher Sprachhöhe zum Ausdruck kommen, wie zu trennen ist zwischen "Grundwissen" und Inhalten, die als "spezieller Stoff « gelten können, das ist eine "Crux«, mit der auch Cochlaeus sich auseinanderzusetzen hatte (43). Keiner dieser Teilbereiche des weiten Felds Musiklehre ist dazu in der Lage, den jeweils anderen die Daseinsberechtigung abzusprechen, im Gegenteil: Die Spannungen, die durch die Vermittlung dieser Aspekte entstehen, auszuhalten und immer neu zu verhandeln, das macht die Musiklehre seit Jahrhunderten aus. Wenige Quellen machen das so deutlich wie die Musica des Johannes Cochlaeus in ihrer komplexen Entstehungs-, Editions- und Rezeptionsgeschichte, die nun erstmals in einer überschaubaren Form zugänglich und umfassend erschlossen ist. Dass Klaus-Jürgen Sachs die Forschung mit dieser bis ins Detail hinein vorbildlich ausgeführten Edition und einer kenntnisreichen Studie ein weiteres Mal substantiell bereichert hat, das verdient - darin kann man den Reihenherausgebern Thomas Ertelt und Heinz von Loesch nur zustimmen - »neben unserem herzlichsten Dank auch den allergrößten Respekt« (vii).

Felix Diergarten

8 „Etsi ars atque virtus circa difficilia versantur, teste philosopho, cum tamen queque difficilima videntur iis, qui horum ipsorum ignari sunt, opere precium esse duxi, docentes pro frugi addiscentium commodo varia attentare secundum variam atque inequalem ingeniorum capacitatem« (200).

9 Siehe das in Anm. 8 wiedergegebene lateinische Original.

10 »sed etiam pro vitando scandalo, ne scilicet stetur in choro, muti more, aut si canatur discorditer cum aliis, astantium ledatur auditus« (200). 


\section{Literatur}

Cochlaeus, Johannes (1970), Tetrachordum Musices. Introduction, Translation and Transcription, hg. von Clement A. Miller, o. O.: American Institute of Musicology.

Fenlon, lain (1994), „Heinrich Glarean's books", in: Music in the German Renaissance. Sources, Styles, and Contexts, hg. von John Kmetz, Cambridge: Cambridge University Press, 74-102.

Judd, Cristle Collins (2000), Reading Renaissance Music Theory. Hearing with the Eyes, Cambridge: Cambridge University Press.

Kleinertz, Rainer / Christoph Flamm / Wolf Frobenius (Hg.) (2010), Musik des Mittelalters und der Renaissance. Festschrift Klaus-Jürgen Sachs zum 80. Geburtstag, Hildesheim: Olms.

Mai Groote, Inga (2013), "Studying Music and Arithmetic with Glarean: Contextualizing the Epitomes and Annotationes among the Sources for Glarean's Teaching", in: Heinrich Glarean's Books. The Intellectual World of a Sixteenth-Century Musical Humanist, hg. von lain Fenlon und Inga Mai Groote, Cambridge: Cambridge University Press, 195-222.
Sachs, Klaus-Jürgen (1974), Der Contrapunctus im 14. und 15. Jahrhundert. Untersuchungen zum Terminus, zur Lehre und zu den Quellen, Wiesbaden: Steiner.

- (1984), „Die Contrapunctus-Lehre im 14. und 15. Jahrhundert«, in: Hans Heinrich Eggebrecht / F. Alberto Gallo / Max Haas / Klaus-Jürgen Sachs, Die mittelalterliche Lehre von der Mehrstimmigkeit, Darmstadt: Wissenschaftliche Buchgesellschaft, 161-256.

- (2002), De modo componendi. Studien zu musikalischen Lehrtexten des späten 15. Jahrhunderts, Hildesheim: Olms.

Schwind, Elisabeth (2009), Kadenz und Kontrapunkt. Zur Kompositionslehre der klassischen Vokalpolyphonie, Hildesheim: Olms.

Weiss, Susan Forscher (2010), „Vandals, Students, or Scholars? Handwritten Clues in Renaissance Music Textbooks", in: Music Education in the Middle Ages and the Renaissance, hg. von Russel E. Murray, Susan Forscher Weiss und Cynthia J. Cyrus, Bloomington (IN): Indiana University Press, 207-246. 